\title{
Façonner le corps, régénérer l'individu et danser la nation
}

Marie-Pierre Gibert

\section{(2) OpenEdition \\ 12 Journals}

Édition électronique

URL : http://journals.openedition.org/pa/289

DOI : $10.4000 /$ pa.289

ISSN : 2273-0362

Éditeur

Université Lumière Lyon 2

Édition imprimée

Pagination : 189-219

ISBN : 1634-7706

ISSN : 1634-7706

\section{Référence électronique}




\section{Façonner le corps, régénérer l'individu et danser la nation}

Marie-Pierre Gibert

Université Lumière Lyon 2, CREA

Née officiellement en 1944, inconnue du grand public en dehors d'Israël quoique largement pratiquée par des Juifs et des non-Juifs tant en Israël que dans plus de trente pays, de l'Asie à l'Europe en passant par les Amériques', s'enrichissant tous les ans de plusieurs centaines de nouvelles danses ${ }^{2}$, la danse populaire israélienne (Rikoudéi Am en hébreu, littéralement « les danses du peuple ») semble toujours bien vivante en ce début du XXIe siècle ${ }^{3}$.

Mai 1944. L'immigration juive en Palestine est de plus en plus intense depuis la dernière décade du XIXe siècle. La seconde guerre mondiale ravage l'Europe, l'horreur des camps d'extermination est peu à peu révélée. Une vaste rencontre de danse se tient au kibboutz Dalia, dans la Palestine sous mandat britannique. 200 danseurs sont présents, 3500 spectateurs les rejoignent au fil des deux journées que dure la rencontre, une dizaine de danses dites "israéliennes" (eretz israeli) élaborées pour l'occasion sont présentées par leurs créatrices, et enseignées aux autres danseurs présents qui se chargeront à leur tour de les transmettre à d'autres. Le mouvement des Rikoudéi Am est lancé.

Mais de quoi s'agit-il exactement? Et pourquoi cette forme dansée, ici, dans un numéro portant sur les présences au corps, dans une section sur

1 Voir la liste non exhaustive des offres de cours et de stages de danse israélienne disponibles de part le monde sur le site australien www.israelidances.com

2250 nouvelles danses en 2013, presque 300 par an en moyenne depuis 2000 - Calcul mené grâce au catalogue des danses répertoriées sur le site www.israelidances.com

${ }^{3}$ Quelques chiffres supplémentaires pour décrire l'ampleur et la diffusion de ce phénomène : au début des années 2000, il existait en moyenne, par jour, une soixantaine de soirées de pratique de danse populaire israélienne (harkadot), qui rassemblent entre 100 et 700 personnes par soirée - le pays ayant une population totale de 8M d'habitants; en 2014 on en compte désormais plus d'une centaine certains soirs, et presque 500 par semaine ; presque chaque ville ou municipalité possède au moins une troupe de danse populaire israélienne (composée de plusieurs groupes d'âge), sans compter de nombreux groupes de danse dans les lycées ou les universités. En 1973, Michman note qu'en Israël, « la danse populaire israélienne est la forme la plus commune d'activité dansée » (1973 : 34) ; en 1990, 8\% de la population juive de plus de 14 ans pratiquait cette danse lors des harkadot, tandis que $40 \%$ des offres d'activité culturelle étaient des offres de chant ou de danse populaire israélienne (D. Ronen, 1994 : 123 et interview 1998). 
l'immigration ? En quoi l'analyse de la création et du développement d'une pratique dansée spécifique peut-elle intéresser l'anthropologie sociale de manière large?

La réponse suit trois fils qui vont se nouer au cours de cet article : analyse quasi clinique de la construction d'un État-nation à travers l'élaboration d'une pratique corporelle; réflexion sur le rapport au corps et l'incorporation ; question des modes «d'intégration » des migrants dans une nouvelle société4.

Sous la conjonction d'un antisémitisme récurrent ${ }^{5}$ et $\mathrm{du}$ constat de l'impossibilité, par et pour les populations juives européennes, d'être totalement acceptées par le reste de la société tant en Europe de l'Ouest qu'en Europe de $\mathrm{l}^{\prime} \mathrm{Est}^{6}$, se développe la, ou plutôt les, pensées sionistes au cours du XIXe siècle, pensées qui prennent un tournant nouveau avec T. Herzl (18601904) et le Premier Congrès sioniste (Bâle, 1897). Je n'entrerai pas ici dans la complexité de cette pensée et de ses différentes tendances ${ }^{7}$ et me contenterai d'en décrire les points communs et éléments pertinents nécessaires à la compréhension de cet article. Des premiers temps de la pensée sioniste à l'époque de la création des Rikoudei Am (danses populaires israéliennes), l'un des objectifs explicite de plusieurs tendances sionistes est celui de (re)construire un État-nation séculier, Israël ${ }^{8}$, qui deviendrait le lieu de rassemblement des Juifs éparpillés en diaspora depuis bientôt 2000 ans et n'ayant plus gouverné de territoire autonome depuis lors ${ }^{9}$. A la volonté $\mathrm{d}^{\prime}$ abord idéologique de certains d'entre eux de regagner ce qu'ils considèrent comme «leur patrie », s'ajoute pour de nombreux Juifs d'Europe une réponse pragmatique aux dangers de l'antisémitisme.

Cette (re)construction d'un État israélien s'accompagne de la volonté de «normalisation» de cet État-nation en devenir, selon les critères des nationalismes européens de l'époque : un peuple, une terre, une langue, une culture (Renan 1882, Anderson 1983, Gellner 1999, Thiesse 1999). Or les Juifs

\footnotetext{
${ }^{4}$ Je tiens à remercier ici J. B. Ingber qui, sans le savoir, m'a mise sur la voie de cette réflexion sur le corps dans la création des Rikudéi Am grâce à son article «Vilified or glorified » (2000).

${ }^{5}$ Des brimades quotidiennes aux pogroms, de l'Affaire Dreyfus à la Shoah. Pour un travail historique et politiste complet et critique sur la création de l'État d'Israël et sa genèse, voir notamment Dieckhoff (1993) auquel il sera beaucoup fait référence ici.

${ }^{6}$ Voir le courant des Lumières juives (Haskala) qui ont prôné l'assimilation et la citoyenneté des Juifs à part entière. Ce courant naît à la fin du XVIII siècle, en Allemagne, sous l'impulsion de Moses Mendelssohn, et va se propager dans toute l'Europe Centrale et Orientale au cours du siècle suivant.

7 Parmi les différentes tendances du sionisme, se trouvent en particulier le sionisme politique (M. Nordau, T. Herzl), le sionisme culturel (Ahad Ha-Am) et le sionisme pragmatique. Il existe également une branche appelée "sionisme religieux » qui rappelle que l'un des points communs de toutes les autres branches est celui d'être un mouvement séculier.

8 C'est en particulier le cas du sionisme politique; les tenants du sionisme culturel ne souhaitent en revanche pas l'établissement d'un État, mais d'une entité non politique qu'ils nomment « foyer culturel».

${ }_{9}^{9}$ Sur le rapport des Juifs au politique, voir Dieckhoff (1989).
} 
ayant vécu plusieurs siècles dans des environnements socio-culturels extrêmement divers ${ }^{10}$, cette construction nationale doit passer par la création d'une culture unique, la culture eretz israeli (litt. « de la terre/du pays d'Israël »), qui permettra d'unifier ces différences. Cette normalisation de l'État-nation se loge en outre dans l'élaboration d'un « Homme Nouveau »11, ou « Nouvel Hébreu », un juif « régénéré » 12 dont le corps serait façonné pour répondre aux différents enjeux mis en avant par les pensées sionistes de l'époque: lutte contre les stéréotypes antisémites, construction/ "renaissance » d'un pays, unification d'une nation, etc. ${ }^{13}$ Combinant ces deux attentes du sionisme (culture nouvelle et transformation des corps), se crée une nouvelle pratique dansée, forme unique, unissant les citoyens de l'État-nation en devenir, et gommant les disparités issues des siècles vécus en diaspora aux quatre coins du monde. Ainsi, en construisant une nouvelle forme dansée, il s'agit à la fois de refaçonner les corps des Juifs et de les ré-unir dans une culture et une nation commune.

Revenir sur l'histoire de la création et du développement de cette forme dansée jusqu'à la situation actuelle devrait donc permettre d'envisager sous un jour nouveau la manière dont la construction du corps humain selon un modèle spécifique est au cœur d'enjeux politiques allant bien au-delà de la « simple » question des pratiques artistiques.

Dans une première partie, je $\mathrm{m}^{\prime}$ arrêterai sur les enjeux et les processus de construction d'une culture désirant unir et unifier tous les Juifs, mêlant danse et migration. La seconde partie effectuera un retour en arrière historique afin de comprendre pourquoi les réflexions autour du façonnage des corps sont centrales dans le sionisme. Une troisième partie reposera la question de la transformation corporelle sous l'angle plus théorique d'une anthropologie prenant les pratiques dansées comme objet de recherche.

Cette situation, pour spécifique qu'elle est, nous permet de réfléchir, audelà du cas particulier de la danse populaire israélienne, à la nature dynamique des pratiques culturelles en tentant de rompre avec les débats stériles en termes de «tradition » ou d' «authenticité ». Ainsi, m’inspirant des

10 Europe de l'Ouest, Europe de l'Est, Amérique du Nord, Amérique du Sud, Afrique du Nord, Afrique de l'Est et du Sud, Moyen-Orient et Péninsule Arabique, Asie du Sud Est.

${ }^{11}$ La notion d' «Homme Nouveau » ne nait pas en Europe à cette époque-là, elle traverse déjà le XVIIIe et le début du XIXe siècles. Voir M. Ozouf (1989 : 116-154) et Mosse (1997). Sur la notion de "renaissance » dans les travaux de l'un des penseurs du sionisme, M. Buber, voir Wildmann (2006:30).

12 Comme le montre M. Ozouf dans son travail sur la Révolution Française, c'est avec la notion d' «Homme Nouveau» que les champs sémantiques de la dégénérescence et de la régénération entrent dans de très nombreux écrits des années précédant la Révolution Française (1989 : 128 et suiv.). Cette terminologie sera à nouveau mobilisée à la fin du XIXe siècle, notamment en médecine et dans les écrits sionistes, comme en témoignent les écrits de M. Nordau sur la « dégénérescence » (1892).

13 Pour un travail historique critique sur cette (re)construction, voir en particulier les travaux de Y. Zerubavel. 
travaux fondateurs de Pouillon $(1975,1993)$ et Lenclud $(1987,1994)$ qui montrent comment les discours autour de la tradition sont en fait une forme de lecture du passé au prisme des nécessités du présent, ou ceux d'Hobsbawm et Ranger sur "l'invention de la tradition» (1983), je propose de penser les questions de "transformation » en évacuant le critère de changement/non changement pour envisager les transformations selon un continuum variant en fonction du critère de conscience/volonté versus non conscience/non volonté de transformation. Ainsi, à une extrémité du continuum se trouvent les transformations inconscientes et/ou involontaires. Il peut s'agir par exemple de ce que J. Goody montrait déjà dans son étude des versions de la récitation du bagré (1972) - versions considérées comme identiques par ceux qui les récitent mais apparaissant différentes lors de l'analyse textuelle ; ou des "transformations silencieuses» qu'analyse le philosophe François Jullien (2009) - changements fins invisibles sur le moment mais visibles sur le long terme, tels les processus de vieillissement du corps. A l'autre extrémité se trouvent les transformations volontaires, conscientes, recherchées, telles que celles données à voir dans cet article. Ces transformations sont menées en prenant appui sur - ou contre - des références (origine mythique, stéréotypes, transmetteur, partenaire de danse, etc.), et face à des enjeux implicites ou explicites qu'il nous faut examiner de près. S'ajoute à cette analyse celle des moments de cristallisation de certaines de ces transformations, ces cristallisations pouvant prendre différentes formes ou passer par différents stades: constat, revendication, fixation, reconnaissance, légitimation, normalisation, officialisation, institutionnalisation, etc., qu'il s'agira à chaque fois d'étudier en termes de traitement de la matière et d'action par, et sur, les individus concernés.

\section{CONSTRUIRE UNE CULTURE DANS UNE UNICITÉ DES CORPS ET DES PRATIQUES}

Dès les premières vagues d'immigration juives de l'Europe vers la Palestine à la fin du XIXe siècle ${ }^{14}$, la volonté des nouveaux arrivants nourris des idéaux sionistes de l'époque est de mettre en place les bases de ce qui devra ensuite devenir un État: édification de logements, villes et villages agricoles, asséchage des marais et travail des terres non cultivées, construction $\mathrm{d}$ 'infrastructures routières, etc. mais aussi élaboration progressive de structures organisationnelles pré-étatiques, et poursuite des réflexions

14 L'historiographie israélienne a donné un nom particulier aux vagues d'immigration inspirées du sionisme et s'étalant de la fin du XIXe siècle (1882) à la création de l'État (1948). On parle alors d'aliot (alya au singulier), terme qui vient de l'hébreu «montée » et qui signifie ici la «montée » vers « la terre promise ». A l'exception d'une alya venue du Yémen en 1882, toutes les autres viennent d'Europe. 
politiques en termes de construction d'une «culture " spécifique, la culture eretz israeli.

Cette construction culturelle s'articule principalement autour de trois piliers centraux de l'idéologie sioniste de l'époque ${ }^{15}$. Il s'agit (1) de dépasser (en les oblitérant au maximum) les années passées en diaspora depuis la dispersion des Juifs en 135 ap. JC pour, (2) se concentrer sur la nouvelle expérience de vie en cours d'élaboration : période de "renaissance " d'une patrie juive et d'un « Homme Nouveau ». Pour autant, (3) cette élaboration ne doit pas se faire exnihilo, elle cherche à retrouver les pratiques juives qui auraient existées à "l'époque de la Bible", époque largement mythique prise comme référence dans la réflexion sioniste car considérée comme la dernière période historique au cours de laquelle les Juifs possédaient un pays «à eux »16. Buts cohérents dans la théorie de ce système de pensée, ils sont difficilement conciliables dans la pratique : Comment être à la fois au XXe siècle et au Ier? Comment vivre " au plus proche des Juifs des Temps bibliques» tout en prenant acte des transformations radicales de la vie humaine en vingt siècles, et de l'expérience actuelle vécue par ceux que l'on nomme alors les «pionniers » (khaloutzim) ${ }^{17}$ ? Comment puiser dans le texte religieux de la Bible comme dans un livre d'histoire et un manuel de construction d'un État-nation ? Comment être tous identiques alors que les différentes populations juives ont évolué pendant presque vingt siècles dans des environnements géographiques, politiques, linguistiques et sociaux parfois radicalement différents? Ces questions font écho à celles que se posait justement $\mathrm{T}$. Herzl, $\mathrm{l}^{\prime}$ un des fondateurs du sionisme politique, comme l'indique le titre de son « roman d'anticipation» : Altneuland («Terre ancienne et nouvelle») publié en 1902 et décrivant sa vision de la Palestine future.

En outre, si la situation est politiquement envisagée comme le retour des « exilés diasporiques» (galouyot), il n'en reste pas moins que, en pratique, il s'agit d'individus dont seuls les très lointains ancêtres ont vécu en Palestine, mais qui eux-mêmes viennent y vivre pour la première fois après des siècles de vie dans d'autres environnements socio-culturels ${ }^{18}$. Pour cette raison, je propose de nommer cette situation «diaspora inversée » 19 puisque ce

15 Voir notamment les écrits du chef de file du sionisme culturel, Ahad-Ha-Am (1938 [1894]).

16 Sur ce travail de recherche de références culturelles, mythes et héros nationaux dans la Bible par les créateurs de l'État, voir Y. Zerubavel (1995, 1998), Sternhell (1999). Sur le rapport au politique, voir Dieckhoff (1989).

${ }_{17} C^{\prime}$ est-à-dire celles et ceux qui sont arrivés (ou vivaient déjà) en Palestine avant 1948 et ont participé directement à la mise en place du futur État.

18 A l'exception bien sûr des membres de la petite population juive étant demeurée sur ce territoire pendant tous ces siècles.

${ }^{19}$ A ma connaissance, cette expression n'est pas utilisée par d'autres auteurs. On trouve en revanche le terme de "double diaspora» pour qualifier ce que vivent certains groupes subissant deux diasporas successives, mais cela ne me semble pas rendre suffisamment compte du paradoxe qui nous intéresse ici. Pour sa part, la politiste D.R. Divine exprime cette tension en intitulant son ouvrage Exiled in the Homeland (2009). 
phénomène atypique de migration est vu comme un retour, mais largement vécu, en pratique, comme une immigration. Or ce paradoxe et cette ambivalence jouent un rôle important dans ce qui va suivre.

C'est dans ce cadre que l'on doit envisager la mise en place des Rikoudéi Am, "ancienne-nouvelle" forme de danse répondant aux trois dimensions de l'idéal sioniste en permettant à la fois un travail des corps et des savoirs de la population juive en Palestine. Dès les années 1920, sont apparues des expériences de créations dansées juives, mais elles restent cependant les initiatives d'individus ou de petits groupes distincts souhaitant participer à leur échelle à la construction d'un «foyer culturel» juif en Palestine. C'est le cas par exemple du «Ballet Biblique » de Rina Nikova ${ }^{20}$, du travail de Baruch Agadati (Manor 1976, Aldor 1999), ou même du concours de danse pour la danse de spectacle «la plus eretz israeli en 1937 (Spiegel 2000, 2013), mais aussi et surtout des habitants de différents kibboutz souhaitant recréer les célébrations hébraïques antérieures à la vie en diaspora (Shavit \& Sitton 2004). L'organisation de ces célébrations en kibboutz illustre parfaitement la triple volonté sioniste énoncée précédemment: ne voulant plus célébrer les fêtes associées à un passé diasporique que l'on veut oublier, les habitants des kibboutz vont chercher dans la Bible les occasions de réjouissance des anciens Hébreux et vont s'en servir comme base à l'édification d'un nouveau calendrier festif tourné désormais vers les célébrations agraires et non plus vers les commémorations des épreuves vécues par le peuple Juif en exil21. L'historienne de la danse J. Brin Ingber (1979), et plus récemment Shavit \& Sitton (2004) et Spiegel (2013), montrent alors comment la plupart de celles car ce sont d'abord des femmes - qui ont ensuite été au cœur de la création et du développement des Rikoudei Am ont auparavant travaillé de manière ponctuelle ou régulière à l'élaboration de célébrations de ce type à l'échelle d'un kibboutz, puis comment l'une d'entre elles, Gourit Kadman, particulièrement visionnaire et investie dans la construction du pays à une échelle plus large, imagine de conjuguer ces initiatives individuelles dans un mouvement collectif. Invitée à élaborer et coordonner une célébration de la fête de Shavouot au kibboutz Dalia en 1944, elle propose de transformer ce qui ne devait être qu'une journée de réjouissance pour les membres de ce kibboutz en un large rassemblement de créateurs, danseurs et spectateurs venus de toute la Palestine, faisant de cette rencontre à la fois un état des lieux et une vitrine de ce qui existe dans le pays en termes de danse en ces débuts des années 1940, et un premier épisode de réflexion et d'enseignement autour de

\footnotetext{
${ }^{20}$ Voir article anonyme extrait du journal Illustrated (3.4.1948) ; Spiegel (2013 : 116-119).

21 Trois festivals ou pèlerinages apparaissent dans la Bible - qui pendant l'Exil étaient essentiellement associés à des événements religieux et historiques - et sont repris dans leur version "agraire» par les habitants des kibboutz: Souccot (Fête des récoltes, automne), Shavouot (Fête de la Moisson ou Offrande des Prémices; printemps) et Pessah (Fête du printemps). En outre, une autre fête liée à la nature est célébrée : Tou biChvat (littéralement « 15e jour du mois de chevet »), appelée aussi Nouvel An des arbres.
} 
la nouvelle forme de danse à construire ${ }^{22}$. Ainsi, si l'on analyse ce qui se passe lors de ce rassemblement, il apparaît que toutes les composantes de ce qui va devenir ensuite la forme de danse que l'on connaît jusqu'à aujourd'hui sous le nom de Rikoudei Am sont déjà visibles dans cet événement fondateur :

Travail explicite, réfléchi et organisé (réunions, débats, commissions de décision, institutionnalisation de la création, de l'enseignement et de la diffusion, etc. ${ }^{23}$ de création et de développement d'une forme dansée qui permettra l'unification des corps et des pratiques culturelles pour parvenir à faire des futurs israéliens « une nation qui danse » (Am roked, titre de l'ouvrage de G. Kadman, 1969) ;

Modalités d'enseignement et de dissémination par apprentissage en cascade tant en Palestine/Israël qu'à l'étranger24 ;

Double modalité de pratique de ces danses: spectacle destiné à être regardé, et pratique collective non spectaculaire (harkada) ;

Standardisation du format des danses qui composent le répertoire des Rikoudei Am: chaque entité "danse " (rikoud) correspond à une chorégraphie spécifique de quelques minutes, constituée d'une ou plusieurs séquences fixes de mouvements et de gestes répétés ad libitum, effectuées sur une mélodie spécifique, le tout étant parfaitement identifié par un nom de danse, un nom de chorégraphe, une date de création, et éventuellement un nom de musicien ${ }^{25}$.

Mais qu'en est-il du matériel dansé lui-même? De quoi sont donc faites, concrètement, ces «nouvelles » danses israéliennes, et comment permettentelles d'atteindre le but fixé d'unification culturelle et corporelle?

Si la dizaine de danses présentées lors de ce premier rassemblement de Dalia 1944 est le fruit de réflexions éparpillées de créatrices n'ayant pas encore partagé discussions, débats et démarches à adopter, cette réflexion et sa mise en œuvre concrète se structurent dès cette première rencontre. Les trois

${ }^{22}$ Le compte-rendu établi par l'une des participantes au premier cours d'instructeurs qui suivit cette rencontre est extrêmement utile pour saisir les questionnements qui surgissent à cette étape : "The first course for folk dance (instructors) was dedicated to the search for Israeli folk dance. The question was, what shall replace the non-Israeli dances danced in the dininghalls of the kibbutzim. Just as in Israeli song, the direction is to face eastwards." Bulletin du kibboutz de cette participante, paru en février 1945, cité par Friedhaber (1987/88: 36).

${ }^{23}$ Comme le note l'historien T. Segev, ce travail de réflexion semble avoir été une démarche générale dans l'Israël des premières décades, et non seulement pour la danse : " Les Premiers Israéliens se livraient volontiers à des débats introspectifs où ils tentaient de façonner leur identité nationale et culturelle de Juifs et d'Israéliens. Ce n'était pas chose aisée. L'actualité engendrait sans cesse de longues discussions qui, toutes, les ramenaient inéluctablement aux questions fondamentales : 'Qui sommes nous ? Que voulons nous être'. » (1998: 324).

${ }^{24}$ Quelques couples de danseurs/danseuses de différentes entités (kibboutz, moshav, « centre de loisir », régiment militaire, etc.) sont ainsi invités à une séance d'apprentissage des nouvelles danses, puis ils repartent chacun pour transmettre cet enseignement à tous les membres du kibboutz / moshav / centre / régiment / etc.

${ }^{25}$ Pour se faire une idée de ce que cela peut donner en pratique, consulter la base de données des danses sur le site de Israeli Dances.com (voir supra). 
dimensions de l'idéologie sioniste présentées plus haut sont au cœur de ces échanges. D'abord, les précédentes recherches menées par les créatrices dans le texte biblique sur les occasions de danser sont mises en commun et discutées. Mais s'il est possible d'y identifier des moments, des contextes, des personnages, et éventuellement des lieux de danse, ce texte ne renseigne quasiment pas sur la dimension corporelle (positions) et encore moins sur la dimension kinésique (mouvements effectués) de ces pratiques dansées pensées comme extrêmement anciennes ${ }^{26}$. Et comme le Judaïsme interdit les reproductions picturales des corps humains ${ }^{27}$, aucune iconographie ne peut être sollicitée dans la reconstruction d'une forme dansée «biblique ». Celles qui seront nommées «les pionnières de la danse israélienne ${ }^{28}$ se tournent alors vers le présent : que trouve-t-on en termes de danse dans la Palestine des premières décades du $X X^{e}$ siècle? Pratiques dansées des populations arabes (Chrétiens, Musulmans et Druzes) ou juives vivant sur ces terres depuis plusieurs siècles, ou des Tcherkess (Musulmans du Caucase) vivant dans quelques villages de Galilée depuis le milieu du XIXe siècle; pratiques dansées apportées par les populations juives arrivées plus récemment d'Europe de l'Est (Russie, Pologne, Lituanie, Roumanie, etc.), d'Europe de l'Ouest (Allemagne, France) ou du Yémen ${ }^{29}$. Les répertoires dansés de ces différentes populations juives et non-juives sont alors étudiés et disséqués, et des éléments de natures très différentes en sont extraits : ici un rythme $(7 / 8$, $4 / 4,3 / 4)$, là une configuration de danseurs (cercle fermé, file, couples), ailleurs un "pas » spécifique (succession fixe d'appuis), une manière de se tenir entre danseurs, etc. Ces différents éléments constituent alors un matériel multiple, renvoyant à divers plans du corps en mouvement, dans lesquels les créatrices vont puiser pour construire leurs propres séquences dansées. S'y ajoutent un ensemble d'images que les chorégraphes souhaitent insérer dans leurs créations : celle d'un corps sain, dynamique et tonique, celle de la fierté d'être en train de construire un pays et de nouvelles formes de vies, etc. images qui se traduisent notamment par le fait que danseurs et danseuses

${ }^{26}$ Contrairement à ce qui se passe à peu près à la même époque en Inde (Leucci 2008). A. Shay souligne lui aussi la pratique courante, dans les constructions nationalistes par la danse, de légitimer une pratique dansée actuelle par une référence à un passé lointain et imprécis (1999: 35).

27 Le respect du Deuxième Commandement entraine la quasi inexistence de toute représentation figurée: "Tu ne te feras point d'idole, ni une image quelconque de ce qui est en haut dans le ciel, ou en bas sur la terre, ou dans les eaux au-dessous de la terre » (Exode XX, 4).

28 La mobilisation du terme "pionnière » pour désigner ces femmes est révélatrice de l'importance qui est attribuée à leur travail. Elles sont considérées comme construisant pleinement le pays en devenir.

${ }_{29}$ Après 1948, viendront les Juifs du bassin méditerranéen, les Juifs des Amériques, les Juifs $\mathrm{d}$ 'Asie (Inde, Chine), puis les Juifs Ethiopiens, et plus tard encore arrivera une nouvelle vague de Juifs venus de l'ex-URSS. Les répertoires dansés respectifs de ces différents groupes sont jusqu'à aujourd'hui inégalement intégrés dans les rikoudéi am. 
«bondissent», «se tiennent droits et fiers », dansent souvent pieds nus, sont vêtus de costumes légers et pratiques, etc. Ainsi G. Kadman, revenant sur les spécificités de la danse israélienne, souligne que :

l'humeur et l'expression de l'exécution, et les mouvements du corps qui l'accompagne (...) reflètent le caractère spécifique de la jeunesse israélienne et l'impact des évènements historiques récents (comme la lutte pour l'indépendance) (1972c: 33) ${ }^{30}$.

Certains danseurs se souviennent de cette époque et de leurs difficultés dans l'apprentissage de certains éléments corporels tant ils contrastaient avec leur propre corporéité en construction. C'est le cas notamment pour l'apprentissage de la succession de mouvements nommés « pas yéménite »31:

En fait j'ai appris le Pas yéménite de Rachel Nadav lors d'un festival de Shavouot, peut-être en 1949. Je me souviens que c'était extrêmement difficile pour nous de comprendre, car nous étions habitués à faire de très grands pas et de larges bonds. C'est comme cela que les choses ont changé pour moi au début - passer de quelque chose de très fier et plein de bravoure à une calme joie venant du cœur (Y. Ashriel, in Ingber, 1974 : 46).

Selon les créatrices des Rikoudéi Am, c'est précisément dans le travail de déconstruction-reconstruction que se loge la nature «nouvelle» et « israélienne » des danses ainsi créées. En effet, il ne s'agit pas de présenter telle ou telle spécificité régionale dans le produit fini de la nouvelle forme dansée, mais au contraire d'extraire des différents répertoires sollicités (juifs et non juifs) une sorte de « supra-hébraïté » qui transcenderait les spécificités des différents groupes vivant et/ou arrivant en Israël. Répertoires juifs et nonjuifs, car dans ces premières décades, la référence constante au passé mythique de la Bible se traduit également par l'intérêt particulier des créateurs de la culture israélienne pour les populations arabes autochtones ainsi que pour les Juifs yéménites, populations toutes deux regardées comme ayant potentiellement conservé dans leurs pratiques actuelles ce qui se serait passé pendant ces «Temps bibliques » de référence ${ }^{32}$ :

Il faut rester un moment silencieux, et dans le silence essayer de se libérer de toutes les impressions et les sons dans lesquels vous avez été englouti en diaspora... Le cri sauvage que l'on entend dans ce pays lors d'un mariage arabe

\footnotetext{
${ }^{30}$ La traduction française des citations est de moi, sauf indication contraire.

31 Voir Gibert (2007).

32 Mentionné par Pataï dès la fin des années 1940 pour d'autres domaines culturels : «In Palestine, what the Arabs lacked in higher standards was made up in the romantic admiration of the first Jewish settlers for the indigenous inhabitants of their much coveted ancient homeland $(1947: 19)$. Sur le regard porté sur les Juifs yéménites comme « la préservation du Judaïsme pré-diasporique », voir Weingarten cité par Parfitt (1996 : 11).
} 
est plus significatif pour l'art hébreu du futur que les airs européens officiels, et les danses de nos bâtisseurs de routes sont plus importantes que les danses étrangères les plus modernes. Nous devons apprendre des Sépharades et des Yéménites (Y. Karni, poète et journaliste, 1922, cité par Hirshberg, 1997 : 7).

Mais tandis qu'ils reçoivent ainsi une sorte d'adoubement symbolique pour ce que leurs pratiques musicales ou dansées représentent d' « ancestralité », ces groupes non-européens sont en même temps, et paradoxalement, fortement encouragés à abandonner la pratique de leurs spécificités culturelles en tant que telles, celles-ci devenant de simple réservoirs d'éléments formels mobilisables par la culture israélienne en construction. Les liens qui unissent dimensions ethnologiques et dimensions formelles des anciennes pratiques dansées sont donc rompus, mettant ainsi en pratique la volonté de rupture avec le passé diasporique, et de nouveaux liens sont reconstruits selon l'optique de la nouvelle culture israélienne. Ce traitement particulier des matériaux corporels se lit clairement dans les commentaires des premières créatrices. Ces dernières insistent tout particulièrement sur le processus de construction, plutôt que sur les éléments, pour fonder cette « israélinité » :

Le fait le plus important n'est pas que nous Israéliens utilisions la debka arabe ou les pas yéménites ou soyons influencés par le paysage. La personnalité de l'artiste est ce qui est le plus important, plus que les pas qu'il utilise, qui ne sont que des moyens d'expression, comme les crayons à dessin sont les outils du peintre. Donner des noms à ces pas ne les décrit pas, pas plus que cela n'explique pourquoi le créateur les utilise ou pourquoi il ajuste ensemble pas et rythmes comme il l'a fait afin de créer quelque chose de tellement convaincant dans sa simplicité et tellement magnifique que c'est accepté immédiatement par un peuple entier (R. Sturman, citée par Ingber, $1974: 16$ ).

Cette volonté paradoxale de recherche de racines et d'ancestralité tout en se débarrassant des références diasporiques explicites se lit dans le parcours et les justifications des premières créatrices. Le récit que fait $\mathrm{R}$. Sturman de sa prise de conscience est particulièrement significatif de ce processus, d'autant plus que l'on retrouve ce récit sous des formes presque identiques dans différents supports ${ }^{33}$, en faisant l'un des mythes d'origine des Rikoudéi Am :

Rivka Sturman, se promenant dans son kibbutz, aurait entendu des enfants qui répétaient pour un spectacle de fin d'année chanter en allemand. Elle se serait alors dit: «Je ne suis pas venue ici, je n'ai pas coupé mes racines en Allemagne et laissé ma famille derrière moi afin de construire une nouvelle vie en Israël [Palestine] pour que mes enfants écoutent des chants allemands!» (R. Sharett, Entretien. Tel Aviv, 1998).

33 La version rapportée ici provient d'une interview avec sa biographe, Rina Sharett, mais l'histoire est également racontée par Rivka Sturman elle-même dans une vidéo de ses danses, ou encore relatée et publiée par Ingber (1974 : 17). 
Ainsi, par le biais de cette nouvelles pratique corporelle et de sa dissémination rapide et complète à toute la population de la Palestine/Israël, il s'agit de transformer corporellement les Juifs, non seulement pour les extraire des conditions physiques et morales vécues en diaspora, mais également pour les couler dans un moule unique faisant disparaître la multitude de leurs provenances géographiques et la diversité de leurs pratiques culturelles. Cette tension paradoxale participe donc largement au processus de «diaspora inversée » développé précédemment. Et plus encore après la création officielle de l'État d'Israël (1948), moment à partir duquel l'immigration juive $s^{\prime}$ intensifie très rapidement ${ }^{34}$. Le projet de " rassemblement des exilés " (kibboutz ha-galouyot) ${ }^{35}$ va alors être au cœur du programme politique du nouveau gouvernement dont D. Ben Gourion est le Premier Ministre. Le principal travail du gouvernement est en effet de faire face à cet afflux massif et extrêmement rapide d'immigrants. Est alors envisagé un processus auquel il est fait référence par les expressions "absorption de l'immigration" (klitat ha-alya) et "fusion des exilés » (mizug ha-galuyot). Cette intégration est envisagée dans une double perspective: pragmatique bien sûr, car il s'agit de résorber rapidement les vastes problèmes logistiques et économiques engendrés par cette augmentation spectaculaire de la population; mais surtout idéologique: procéder à la "fusion» des différents arrivants afin de parvenir au but sioniste ultime de l'unicité de l'Hébreu Nouveau. En pratique, la tâche du gouvernement de Ben Gourion est immense, mais celui-ci compte en premier lieu sur le pouvoir assimilateur de l'armée, de l'école et de l'administration :

A l'intérieur de l'État, les différences entre les divers types de Juifs seront oblitérées par le temps, les communautés ou tribus fusionneront tôt ou tard en une unité nationale et culturelle. L'éducation commune, la langue hébraïque, le service universel dans les Forces de Défense d'Israël, l'établissement d'un standard de vie minimum commun, l'adhésion de travailleurs issus de divers pays et communautés à une fédération d'ouvriers unique, les mariages mixtes entre les différentes tribus, l'action politique commune dans des partis non communautaires, et ainsi de suite, produira un nouveau type de Juif possédant les qualités et les caractéristiques de toutes les tribus d'Israël (David Ben-Gourion, cité par J. Isaac, $1959: 266)$

\footnotetext{
${ }^{34} \mathrm{La}$ « Loi du retour» - dont le premier article stipule que « Tout Juif a le droit d'immigrer en Israël », déjà inscrite dans la Déclaration d'Indépendance, est adoptée par le Parlement israélien en juillet 1950. Ainsi, en quelques années, la population juive va doubler, passant de 650000 habitants juifs en 1948 à 1400000 en 1951.

${ }^{35}$ L'emploi de cette expression biblique (Dt 30, 3-5) est un exemple particulièrement éclairant de ce double travail de sécularisation et de politisation de la Bible dans la situation contemporaine de l'État d'Israël.
} 
L'État va donc prendre en charge l'organisation de l'ensemble de la vie de ces nouveaux arrivants : logement, éducation, santé, activité professionnelle, etc. (Abitbol, 1995 : 45 ; Charbit, 1996 : 143-145), tandis que les Rikudéi Am se chargent de leur assimilation corporelle. La danse offre ainsi une double combinaison : re-création d'un corps et d'une culture uniques, qui permettent à la fois l'intégration des immigrants et l'union de la nation.

Pour autant, malgré ces volontés explicites de faire table rase de tout le passé diasporique pour placer tous les Israéliens sur le même plan, il apparaît en filigrane que se maintient malgré tout un cadre implicite issu des pratiques culturelles d'Europe de l'ouest, c'est-à-dire celles de la quasi totalité des premiers arrivants ${ }^{36}$. Au-delà de la rhétorique d'égalité et d'unicité de "l'Homme Nouveau », la prééminence idéologique de ceux que l'on qualifie ainsi de "pionniers» sur les arrivants plus récents est évidente. E. Cohen parle à ce sujet d'une «domination symbolique de l'establishment pionnier socialiste sur les immigrants de masse » $(1983$ : 111) qui arrivent dès la fin des années 1940. Cette différence de statut transparaît de fait dans les propos tenus par Ben Gourion bien avant la création effective de l'État :

Un pays ne se construit que par le labeur des pionniers. Une immigration de masse ne se propose pas de mission historique; elle va s'établir là où les conditions ont été préparées à l'avance pour son accueil. La préparation du pays et la création des moyens d'intégration de ces masses sont le rôle des pionniers, les pionniers du travail, de la construction, de la science, armés d'une volonté puissante et du sentiment de leur mission historique, formés à la fusion de leur destinée personnelle et de leur mission et prêts à donner leur vie pour l'accomplissement de leurs aspirations. (1915, reproduit dans Charbit, 1998 : 181183)

Ainsi, tandis que les premiers arrivants sont considérés comme les concepteurs de cet État-nation en devenir, les Juifs arrivants après 1948 sont plutôt envisagés comme "main d'œuvre", voire « bénéficiaires » qui doivent accepter et appliquer les règles établies par d'autres en amont, et ce faisant, ne sont pas considérés comme ayant exactement la même valeur dans la construction de l'État-nation, mais plutôt comme de "simples » immigrants sommés de s'intégrer à une société d'accueil étrangère. C'est donc cette nécessité de parvenir à une intégration qui est invoquée pour justifier cette véritable mise sous tutelle culturelle des nouveaux immigrants (E. Cohen, 1983 : 111) : la notion d'«absorption » traduit d'ailleurs bien la volonté d'un groupe d'avaler l'autre, et non de mener un processus à double sens. Or cette intégration est sous-tendue par le sentiment d'une supériorité de la culture et

\footnotetext{
36 Sur cette terminologie, voir supra. La première vague d'immigration venue du Yémen arrive elle aussi en 1882, date de la «Première alya » européenne. Ce fait est très peu connu si bien que, lorsqu'il est question des premiers pionniers, sauf spécification précise de leur origine extra-européenne, ce n'est qu'aux immigrants européens qu'il est fait référence.
} 
du système de valeurs du pionnier vétéran, Juif Européen. Ainsi, quoique les discours officiels parlent de "melting pot», de "renouveau » et de "fusion » complète $^{37}$, le moule ressemble par bien des aspects à la culture européenne.

$\mathrm{Si}$ ce type de domination est loin d'être conscient, et moins encore volontaire, pour beaucoup d'acteurs et d'actrices de la création d'une culture eretz israeli, un bref arrêt sur le parcours de certaines des premières créatrices des Rikoudéi Am permet de mieux saisir l'environnement intellectuel et politique qui peut sous-tendre, parfois seulement implicitement, les réflexions de nombre d'entre elles ${ }^{38}$.

Parmi les cinq principales figures de cette création identifiées par J. Brin Ingber $(1979,2011)$, deux sont nées en Palestine (Yardena Cohen et Sara LeviTanai ${ }^{39}$ ) et trois (Gourit Kadman, Rivka Sturman et Leah Bergstein) viennent $\mathrm{d}^{\prime}$ Europe centrale (Leipzig et Vienne). Toutes cinq font mention d'une forme ou d'une autre d'éducation sioniste reçue dès leur jeune âge, mais aussi de leur imprégnation par d'autres courants intellectuels en vogue en Europe à la fin du XIXe siècle et au début du XXe siècle ${ }^{40}$. C'est le cas notamment de G. Kadman qui explique le rôle qu'a joué son appartenance aux Wandervögel, et à des mouvements de jeunesse juifs inspirés de ce mouvement de jeunesse allemand (Ingber, 1979: 7). Or la philosophie et les activités de ce mouvement font particulièrement écho à ce qui sera au cœur des réflexions de ces femmes lorsqu'elles tenteront de mettre en place une culture israélienne spécifique: recherches de racines vues comme "authentiques " grâce à des collectes de littérature orale (chants, contes, etc.) souvent rurale, encouragement à une vie " plus proche de la Nature », etc., idées très liées aux courants romantiques et folkloristes européens de l'époque. Ainsi, sous la conjonction de ces différentes sources éducatives, la plupart de ces jeunes femmes décident de suivre des études ou une formation (en Europe puis en Palestine) destinée, expliquent-elles, à participer de manière active à la construction du pays (puériculture, enseignement, médecine, etc.). Parmi ces volontés de travailler à la construction du futur État, G. Kadman, alors professeure d'éducation

\footnotetext{
37 Ben Gourion déclare ainsi en 1949: «Cette nation sera composée d'un grand nombre de tribus. Il nous faut fondre les déchets de l'humanité juive dispersés par-delà le monde qui viendront en Israël dans le creuset de l'Indépendance et de la souveraineté nationale. Il nous faut créer un caractère et un style hébreux qui n'ont jamais existé, qui n'ont jamais pu exister au sein de la Diaspora, chez ces gens dépourvus de patrie, d'indépendance et de liberté nationale » (cité par Segev, $1998: 330$ ).

38 Ce travail d'analyse mériterait à lui seul un article entier. On pourra se reporter au vaste travail d'histoire orale et d'archive effectué par J. Brin Ingber $(1979,2011)$.

39 Cette éducation ne prend pas la même forme pour chacune. Par exemple, S. Levi-Tanai, pourtant née dans une famille juive arrivée du Yémen en Palestine un demi siècle auparavant, est élevée dans un orphelinat tenu par des Européens et éduquée dans ce qu'elle nomme "une éducation sioniste patriotique et hébraïque, pas particulièrement religieuse, et certainement pas yéménite » (citée par Ingber, 1979: 24).

${ }^{40} \mathrm{Il}$ ne sera pas fait mention ici d'autres courants articulant également généralité européenne et spécificité sioniste, tels que les utopies communautaires ou l'orientalisme.
} 
physique, mène dès le début des années 1930 une réflexion sur la nécessité de " reconstruire et reformer les corps, qu'ils soient vigoureux et en bonne santé » (Ingber, 1991 : 16). Cette réflexion est résumée dans son introduction à l'une des premières rencontres entre enseignants d'éducation physique qu'elle organise chez elle à Tel Aviv en 1937 :

La responsabilité qui repose sur les enseignants d'éducation physique en Eretz Israel est immense. (...) Devant nous se trouve la tâche principale du sionisme, le développement sain de la nation hébraïque sur sa terre. Notre but est de rendre le corps sain dans ce moment crucial de développement de la nation. Cette tâche est totalement différente pour les autres nations, il s'agit d'être plus proche de la conscience du corps (G. Kadman, citée par Ingber, $2000: 45)$.

Or ce que G. Kadman appelle ici la «tâche principale du sionisme » vient d'une réflexion sioniste plus large sur le corps juif en diaspora dont elle s'inspire dans la suite de son discours :

Dans notre nation, nous n'avions aucune relation avec le corps pendant les nombreuses générations hors d'Israël. Notre corps doit servir d'abri de santé pour l'âme de la personne juive. (...) Notre peuple est physiquement tourmenté, il est nécessaire de changer cette situation. Nous devons ramener notre peuple à son cours original en termes de corps (G. Kadman, citée par Ingber, 2000 : 45).

Dans ces conditions, poursuit-elle en s'adressant toujours aux professeurs d'éducation physique, il est nécessaire que « le peuple du livre construise son propre corps » (Kadman), et l'éducation des corps a un rôle de premier ordre dans ce processus :

C'est pourquoi le besoin d'un mouvement populaire en éducation physique et d'un mouvement de la culture est immense. (...) Nous ne devons pas oublier qu'une large majorité de notre peuple ici dans le Yishuv n'a jamais fait le moindre exercice physique de manière quotidienne. Il est ironique de remarquer que notre travail de construction du pays est rendu plus difficile parce que notre peuple a des valeurs qui ont été dévoyées. Nous faisons également face à un vaste cercle de personnes convaincues, jusqu'à ce jour, que la culture du corps est quelque chose de contraire à l'esprit du judaïsme et qui placent un accent exagéré sur la vie spirituelle, au détriment du corps, ce qui reste une relique des jours du ghetto. Ce qui doit être mené aujourd'hui est un travail obstiné, énergique et tolérant jusqu'à ce que nous soyons parvenus à établir nous même un mouvement populaire unique. Nous devons entreprendre cela immédiatement (G. Kadman, citée par Ingber, $2000: 45-46$ ).

La pensée sur laquelle s'appuient ces phrases de G. Kadman, cette manière de penser la (re)construction du corps comme étape essentielle dans la construction d'une nation et de son peuple, s'inscrit très clairement dans la réflexion menée par le sionisme, à partir du XIXe siècle, sur le corps des Juifs, réflexion avec laquelle cette chorégraphe a été familiarisée dans sa jeunesse 
européenne et qu'elle embrasse ici de manière extrêmement volontariste. Qu'en est-il plus précisément?

\section{FAÇONNER DES CORPS ET RÉGÉNÉRER DES INDIVIDUS}

Lorsque le médecin juif Max Nordau critique en 1892 la Dégénérescence (Nordau, 1894 [1892-1893]) d'une large partie de la population européenne, il est, comme l'écrit G. L. Mosse, «le produit de son époque (1996: 11). En cette fin de XIXe siècle en effet, nombre d'intellectuels et de médecins juifs et non-juifs considèrent que les phénomènes d'urbanisation et $\mathrm{d}$ 'industrialisation, mais aussi le développement de diverses idéologies politiques (socialisme, anarchisme, libéralisme), participent de l'épuisement nerveux et physique des individus qui y sont confrontés, affaiblissant leurs corps et leurs esprits. Ainsi Nordau oppose-t-il "l'homme dégénéré » ${ }^{41}$ à "l'homme normal à l'esprit clair, au penser logique, au jugement net et à la volonté forte (...) des hommes qui se lèvent tôt et ne sont pas fatigués avant le coucher du soleil, qui ont la tête claire, l'estomac solide et les muscles durs » (1894 (2) [1893] : 531-2). Or, alors que Nordau n'inclut pas directement les Juifs parmi les individus dégénérés qu'il étudie, la plupart des traits de dégénérescence considérés comme tels par l'auteur font écho aux nombreux stéréotypes physiques et moraux utilisés dans les discours antisémites de l'époque $^{42}$. Situation paradoxale à première vue, en réalité phénomène extrêmement courant d'internalisation des stéréotypes par la population incriminée, comme le souligne S. Gilman dans son étude séminale sur les représentations externes du « corps du Juif » et leur influence sur celles et ceux qui sont ainsi représentés :

Ainsi un «Juif » serait autant défini par le contexte social que de manière interne. En effet, il me semble que plus le Juif s'identifie aux buts et aux valeurs de la société qui l'entoure et plus il est touché par le pouvoir de telles images. Mais personne de ceux qui s'identifient, positivement ou négativement, avec le terme «Juif » n'est immunisé contre le pouvoir de tels stéréotypes. (...) Mon intérêt n'est pas seulement pour l'image du Juif, mais pour les forces qui façonnent cette image de différence et - le plus important peut-être - comment ceux qui sont étiquetés comme différents répondent à cette construction de leur identité (1991 : 3-4).

L'auteure montre ainsi comment, en cette fin du XIXe siècle où écrit Nordau, le corps juif est envisagé dans de nombreux écrits antisémites (pseudo-scientifiques ou non) comme la quintessence de la dégénérescence et de l'incapacité physique de l'individu citadin, à ceci près que, tandis que ces

\footnotetext{
${ }^{41}$ Voir le résumé fait par Nordau de ce qu'il appelle les «manifestations fondamentales de la perturbation » en conclusion du 2e volume de Dégénérescence [1893].

42 Voir J. Geller (1996).
} 
faiblesses ne sont envisagées que comme "pathologiques » pour l'individu non-juif, elles sont considérées comme étant la nature profonde de l'individu juif (Gilman, 1991 : 52). Or, continue Gilman, face aux accusations pseudoscientifiques de différences physiques radicales entre Juifs et non-Juifs, telles que celles d'avoir une "voix juive », une psyché fragile, un nez particulier, ou des pieds plats, de nombreux médecins juifs du XIXe siècle tentent de désamorcer ces critiques non pas en réfutant leur base (une hérédité physique spécifiquement juive), mais en cherchant seulement à expliquer les raisons sociales de ces phénomènes, laissant ainsi planer le doute sur l'existence réelle d'une altérité irréductible entre Juifs et non-Juifs telle qu'elle est affirmée par les discours antisémites ${ }^{43}$.

C'est dans ce contexte d'incorporation des stéréotypes antisémites que doivent être envisagées les nombreuses références au corps des Juifs faites dès la deuxième moitié du XIXe siècle par Max Nordau et plusieurs autres auteurs juifs. Ainsi, dès les discours qu'il prononce en ouverture des deux premiers Congrès Sionistes (1897 et 1898) ${ }^{44}$, et plus tard dans ses Ecrits Sionistes (1909), Nordau souligne, pour les condamner, la souffrance et la faiblesse physiques et morales des Juifs de part le monde. Selon lui, l'un des enjeux du sionisme est donc de construire un "Nouvel Hébreu », dont le «corps juif musclé » (MuskelJudentum, Nordau 190045) ne porterait plus les stigmates de la diaspora. Cette reconstruction corporelle, appelée « régénération » et "relèvement » par Nordau (Ecrits Sionistes, version française) est envisagée à la fois comme résistance et comme renaissance: il s'agit de lutter contre le pouvoir destructeur de l'anti-sémitisme à travers une lutte physique contre ce que stigmatisent les stéréotypes - petite taille, corps malingre, dos voûté, santé fragile, manque de virilité, etc. (Gilman 1991, Zimmerman 2006, Mosse 1997).

En pratique, deux démarches sont préconisées: le travail physique et le développement de pratiques sportives. Si le travail physique est surtout envisagé dans le cadre du travail de la terre lors de l'installation en Palestine (nous y reviendrons), la seconde démarche passe notamment par la création dès la fin du XIXe siècle de la "Ligue nationale juive de gymnastique » (Jüdische Turnerschaft) en Europe centrale (Wildmann 2006), l'organisation d'une "Journée internationale de Gymnastique juive » lors du 6e Congrès sioniste de Bâle (1903) (Spiegel, 2013: 188 ; Presner, 2007 : 122), puis par la

\footnotetext{
${ }^{43}$ Voir le chapitre de S. Gilman « The Jewish Foot » (1991).

${ }^{44}$ Comme de nombreux Juifs de l'époque (dont T. Herzl), M. Nordau, qui prônait plutôt jusqu'alors une totale assimilation des Juifs dans le reste de la population européenne, va être fortement ébranlé par l'affaire Dreyfus et "[tiré] brutalement [...] de [ses] illusions datant d'un siècle et [rappelé] à la conscience de la réalité » (Nordau, 1936 [1909] : 27-28). Rencontrant T. Herzl, il se rallie au sionisme politique dès le premier Congrès Sioniste (1897).

${ }^{45} \mathrm{Ce}$ terme est souvent traduit de manières différentes. C'est notamment le cas dans la reprise de ce texte de 1900 dans l'ouvrage de Nordau Ecrits Sionistes, dans lequel le traducteur, B. Hagani, propose l'expression « judaïsme musculaire».
} 
création en 1932 des Maccabiah Games (Olympiades juives) à Tel Aviv (Spiegel 2013).

Or cette démarche s'inscrit dans un mouvement qui dépasse largement la seule population juive de l'époque : celui du développement de l'éducation physique et de la gymnastique en Europe de l'Ouest dans une perspective d'accomplissement à la fois de soi et de la nation, et plus particulièrement celui du Turnen en Allemagne ${ }^{46}$. Comme le développe J. Ulman (1965) et Brenner (2006), ce courant débute fin XIX ${ }^{\mathrm{e}}$ mais est réellement mis en place par F. L. Jahn dans les années 1910 qui se nourrit des réflexions et propositions à la fois pédagogiques et nationalistes de Fichte. Pour ces deux hommes, «le but de l'éducation est (...) essentiellement moral. (...) Elle est l'unique moyen de sauvegarder l'indépendance allemande et, par conséquent, de faire triompher la morale » (Ulman, 1965 : 284-5).

Ainsi, si à un premier niveau, l'éducation fichtéenne à pour but d'

apprendre à l'élève à avoir une claire conscience de ses perceptions, d'abord, de ses intuitions ensuite, et poursuivre, en même temps, par des exercices pratiqués selon les règles de l'art, le développement de son corps (Fichte, Discours à la nation allemande, 1807-8, cité par Ulman 1965 : 284),

à un second niveau, cette éducation physique est

condition essentielle de l'éducation morale et patriotique. (...) Fichte voit en elle le moyen grâce auquel une pensée nécessairement abstraite prendra corps. (...) Ainsi l'éducation physique arrive, chez Fichte, à constituer l'instrument irremplaçable d'une action morale qui se traduit par la constitution d'une communauté nationale solide. Elle a gagné un caractère collectif. Les philosophies kantienne et postkantienne, par le détour de notions abstraites, sont à l'origine d'une conception nouvelle de la gymnastique. La gymnastique n'est plus seulement, comme chez Guts Muths [auteur, en 1793 de l'ouvrage Gymnastik für Jugend (La gymnastique pour la jeunesse)], la condition de la santé, voire d'une sorte d'hygiène morale. Elle est devenue l'indispensable instrument $d^{\prime}$ une action morale dont la première démarche sera d'assurer l'existence et l'indépendance d'une communauté nationale (Ulman, 1965 : 285).

On voit ici en quoi cette influence a pu nourrir la réflexion de Nordau et du sionisme des premiers Congrès et, plus tard, celle des créatrices des Rikoudéi $A m$. Le parallèle entre constitution d'une communauté nationale allemande par la gymnastique et reconstruction du peuple juif par l'éducation physique est immédiat, et se poursuivra pendant toute la première partie du $X X^{\mathrm{e}}$ siècle

46 Voir les chapitres que consacre J. Ulman (1965) au Turnen ; l'ouvrage collectif dirigé par Brenner et Reuveni en 2006 ; l'article de Zimmermann dans cet ouvrage qui montre comment l'idéologie allemande articule sport et réaction nationale (2006:13-14); ou encore les travaux de Presner (2007). 
(Zimmermann 2006, Wildmann 2006). Le ton est donné dans les statuts de la Jüdische Turnerschaft dont le but officiel annoncé est de :

Cultiver la gymnastique comme moyen d'élévation physique (Hebung) de la communauté de descendance juive (jüdischer Stamm) dans le sens d'une idée nationale juive (statuts publiés dans le Jüdische Turn-Zeitung nº9-10 (1903), cités par Wildmann $2006: 27)$.

Il ne s'agit donc pas seulement du travail du corps dans sa simple enveloppe charnelle, mais bien de l'ensemble de l'individu, et même au-delà, du collectif que constituerait une nation. De fait, comme le montre A-M. Thiesse lorsqu' elle parle de «corps patriotique » $(1999: 242)$, les phénomènes de construction du corps concernent de nombreux mouvements nationalistes européens dès le XIXe siècle. Cette idée du corps humain comme instrument privilégié de construction sociale est déjà soulignée par M. Douglas plusieurs décades auparavant :

Le corps humain, plus directement que celui de l'animal, est matière à symbolisme. C'est le modèle par excellence de tout système fini. Ses limites peuvent représenter les frontières menacées ou précaires. Comme le corps a une structure complexe, les fonctions de, et les relations entre, ses différentes parties peuvent servir de symboles à d'autres structures complexes. (...) le corps est un symbole de la société, et (...) le corps humain reproduit à une petite échelle les pouvoirs et les dangers qu'on attribue à la structure sociale (2001 [1967] : 131).

Ainsi, dans le cas qui nous occupe ici, en façonnant, par la danse, le corps des Juifs issus de la diaspora, c'est toute la régénération du «corps de la Nation juive » qui est visée. L'idée de «corps collectif juif » est ainsi mise en avant dans les démonstrations de gymnastique en Europe puis en Palestine (Wildmann 2006: 30-32), démonstrations qui passent notamment par l'effectuation d'exercices synchronisés très proches de la danse.

Sont alors englobés dans la notion d' « Homme Juif » ou « Nouvel Hébreu » à la fois l'individu et le groupe, mais aussi l'homme et la femme, reposant ainsi la question des genres de manière décalée. En effet, comme le soulignent notamment Mosse (1997), Boyarin \& Boyarin (2007) et Lacoue-Labarthe (2012), en détruisant les stéréotypes antisémites du juif malingre et faible, cette construction utopique d'un " Nouvel Hébreu » mène aussi à l'élaboration d'un nouveau stéréotype mettant en avant la dimension virile et masculine des individus ainsi valorisés alors même que les discours insistent plutôt sur la dimension égalitaire de cette nouvelle société et mettent en avant le mythe des femmes pionnières (Lacoue-Labarthe 2012). Sachant alors, comme cela a été vu précédemment, que les premiers «créateurs" de la danse populaire israélienne étaient toutes des femmes, un travail sur ce point reste à mener.

Si l'on se tourne à présent vers la question du travail du sol - deuxième démarche préconisée par le sionisme politique pour la transformation des 
corps juifs en « Hébreux nouveaux » - la notion de régénération est à nouveau mobilisée, non seulement pour l'individu et le groupe, mais aussi pour la terre elle-même. Paraphrasant l'idéologie sioniste-socialiste, l'historien A. Dieckhoff parle de «vision fantasmatique d'une Palestine rédimée par le travail agricole» (1993: 74). Cette double dimension de renaissance traverse la plupart des écrits sionistes de différentes tendances, des déclarations lors des premiers Congrès Sionistes à celles des hommes politiques du futur État :

Un peuple ne se libère pas par hasard; sa liberté ne va pas de soi. A fortiori, un pays ne s'obtient ni ne s'édifie par un mécanisme automatique, sans effort de volonté, sans la ferveur pionnière de ses bâtisseurs. (...) Ce n'est ni par l'argent ni par les droits mais par notre labeur que nous obtiendrons notre patrie. Notre pays, nous le recevrons non d'une conférence de paix ni d'une grande puissance; mais des mains du travailleur hébreu qui viendra s'y établir, le ramener à la vie et y renaître lui-même (D. Ben Gourion, 1915, cité par Charbit 1998).

Cette importance accordée au corps et à sa reconstruction rendra l'adoption d'une forme d'activité corporelle telle que la danse d'autant plus facile que celle-ci pourra remplir de front deux fonctions : rendre les enfants vigoureux et les élever dans la connaissance d'une culture spécifique, unique, et pensée comme «bien à eux».

Mais pourquoi, et surtout, comment, la danse peut-elle tout cela? Cette traversée dans la construction et le développement de la danse populaire israélienne, ses sous-bassements théoriques et sa mise en pratique nous amène en effet à interroger plus avant l'articulation entre corps et pouvoir, via le passage par les processus d'incorporation et de monstration que permet notamment le fait de danser. Il s'agit donc dans une dernière partie de nous arrêter plus particulièrement sur la dimension kinésique de ce qui a été analysé jusqu'ici.

\section{QUAND DANSER, C'EST FAIRE47}

Trois processus concomitants et dialectiques me semblent émerger plus particulièrement du travail pensé et/ou mené sur les corps dans la création des Rikoudei Am:

- Faire ensemble pour incorporer quelque chose d'identique pour tous ceux/toutes celles « qui font »- processus d'incorporation ;

- Faire la même chose que l'Autre pour être/devenir comme lui - processus d'identification ;

47 Paraphrase de Quand dire, c'est faire, titre (dans sa version française) de l'ouvrage de J. Austin, How to Do Things with Words, qui analyse la dimension performative du langage (1970 [1962]). 
- Faire autre chose pour être/affirmer une différence - processus de différenciation.

On retrouve ici les processus mis en exergue par F. Barth (1969) dans son analyse révolutionnaire des constructions d'identité ethnique comme production sociale, qui irrigue depuis plus d'un quart de siècle la réflexion constructiviste en anthropologie. On voit en effet se dessiner ici une production de sens qui s'effectue dans la construction et l'entretien de «frontières » entre des groupes. Construction et entretien sont faits par les groupes eux-mêmes, à la fois de manière interne et de manière externe, par le choix d'un certain nombre de traits mis en avant pour affirmer et maintenir une distinction culturelle, ou au contraire par le gommage d'autres traits pour parvenir à une identification, en fonction des enjeux.

\section{Faire ensemble, faire pareil}

Le premier processus agit dans le cadre interne du groupe qui s'identifie comme «juif ». Il s'agit ici de mettre en place un moyen corporel de rendre tous les Juifs pareils, pour en faire des "hébreux nouveaux» puis des Israéliens. Cela passe à la fois par la forme même des Rikoudei Am, et par les discours qui les entourent. Ainsi, le fait que chaque entité «danse » (rikoud) corresponde à une succession fixe de mouvements, que chacun apprend et peut reproduire à l'identique de son voisin permet une exécution en homokinésie et en homorythmie. Selon les créatrices, cette exécution à l'unisson contribue à provoquer chez les danseurs un sentiment d'appartenance très fort :

La hora est devenue symbole de la nouvelle société énergique. Chaque danseur se tient debout épaule contre épaule avec le danseur suivant, chacun constituant un lien symbolique dans un cercle compact, dynamique, égalitaire (Ingber \& Manor, 1998 : 528).

On retrouve ici la double dimension de travail du corps, à la fois celui de l'individu dansant avec d'autres, et celui du collectif plus abstrait de la nation. Ce sentiment d'unité est alors renforcé par le fait que l'on sait ces mêmes danses en train d'être reproduites exactement de la même manière ailleurs dans le pays, et dans le monde.

Je suppose que la hora était vraiment la danse israélienne de cette époque - pas de manière traditionnelle, puisqu'elle vient de Roumanie, mais de manière réaliste. Elle était si efficace comme affirmation significative pour nous. On pouvait venir de n'importe quel pays de la Diaspora et se joindre à l'esprit de la société Israélienne en dansant la hora. Elle était reconnue comme le moyen d'exprimer l'enthousiasme de construire le pays tous ensemble. La hora était une manière de célébrer cette joie de danser unis dans notre nouvelle patrie (R. Sturman, in Ingber, 1974 : 16). 
Cette force de la dimension imaginée correspond bien à ce qu'analyse $\mathrm{B}$. Anderson (1983) lorsqu'il montre le rôle fondamental de l'imagination dans la construction de communautés et d'États-nations. Poursuivant cette réflexion lorsqu' elle se penche sur la «fabrication de la tradition nationale israélienne » (1995), l'historienne Y. Zerubavel, souligne combien «la mémoire collective est prouvée à travers des formes multiples de commémoration : la célébration d'un festival communautaire, le récit d'un conte, la participation à une cérémonie commémorative, ou l'observance d'une fête » (1995:5).

Or, ajoute-t-elle, il ne s'agit pas seulement d'une démonstration de quelque chose qui existerait déjà, mais bien de processus susceptibles de générer du nouveau :

A travers ces rituels commémoratifs, les groupes créent, articulent, et négocient leurs mémoires partagées d'événements particuliers. L'exécution de rituels commémoratifs permet aux participants non seulement de ranimer et d'affirmer des mémoires anciennes du passé, mais aussi de les modifier (1995 : 5).

Cette analyse de Zerubavel nous ramène à la discussion entamée en introduction sur la nécessité d'analyser les transformations au prisme du critère de la volonté de changement, et de les articuler aux enjeux en action.

\section{Faire comme l'Autre, s'identifier à lui/elle}

Les processus d'identification à l'œuvre ici se déploient le long d'un axe allant du processus volontaire d'un groupe sur lui-même (faire un corps juif musclé; fédérer une collectivité juive; créer une forme de danse) à l'assujettissement d'un groupe à un autre (Juifs non européens / «pionniers»). Ainsi, lorsqu'il s'agit, pour la gymnastique puis pour la nouvelle danse israélienne, de transformer les corps juifs, la volonté des idéologues, des praticiens et des danseurs est commune: rendre ces corps identiques à ceux des Autres, les non-Juifs. En effet, les différences physiques reprochées aux Juifs - et par ricochet ressenties par eux - étant perçues comme un problématique marqueur de différence, devenir musclé et sûr de sa force permettrait de réduire, voire d'annuler, la différence entre Juifs et non-Juifs, menant ainsi à la normalisation recherchée. Ainsi, écrit Nordau,

dans un milieu qui affecte de mépriser les capacités intellectuelles, parce que ne les possédant pas au même degré que les Juifs; qui, par contre, fait parade de ses qualités physiques, parce que croyant en être mieux pourvu que le Juif, il serait de la plus haute importance pour notre situation, de pouvoir chasser nos calomniateurs hargneux de leurs derniers retranchements et d'arriver à les forcer à reconnaître - même contre leur gré - ce fait, que nous les égalons aussi bien comme gymnasiarques que comme intellectuels (1936 [1909] : 117).

Mais, poursuit Nordau, cette pratique physique n'est pas seulement destinée à transformer les corps, elle doit aussi renforcer le moral des Juifs : 
Peu m'importe d'ailleurs que les antisémites aient une meilleure opinion de nous. Ce que ces gens-là pensent de nous, nous est indifférent. Mais il nous faut considérer que le Juif, dispersé, est particulièrement sensible à l'opinion non-juive, et n'estime que ce que son entourage non-juif reconnaît comme ayant de la valeur. Si le Juif se voit consacré et si possible admiré comme gymnaste, comme escrimeur, etc., la bonne opinion qu'il prendra de lui-même l'aidera plus encore à se relever, que la conscience de la sureté, de la force, que ses exercices de gymnastique auront produit en lui. Cet effet de l'éducation physique a pour nous une importance à laquelle il convient de s'arrêter (Nordau, 1936 [1909] : 117-118).

Ce qui se lit en filigrane dans ces mots de Nordau, c'est la volonté de légitimité que pourraient acquérir les Juifs en faisant de la gymnastique, et l'espoir d'être reconnus comme des pairs par les non-Juifs dont ils partageraient désormais les activités, et par extension, le statut de membre à part entière des sociétés européennes dans lesquelles ils vivent.

A l'autre extrémité de cet axe se trouve la manière dont cette volonté de devenir l'Autre peut au contraire être imposée à de tierces personnes. Dans ce cas, la transformation corporelle devient contrôle des corps, comme le souligne C. Detrez tissant ensemble les travaux de M. Foucault (1975) et de G. Vigarello (1978) :

Le corps redressé est ainsi celui "qui marche droit», pourrait-on dire, tant les mots sont pris dans un tissu métaphorique. (...) En redressant les corps, on vise également l'âme, dans ce souci polymorphe de «correction»: correction du langage, des attitudes, par le polissage des aspérités, des déviances et déviations, qui, d'un paysan, fera un élève ou un soldat "poli ». Dans la droiture se jouent ainsi également une norme physique et un jugement moral. Nombreux sont ainsi les termes qui, touchant au corps, manifestent cette sédimentation, ces "faisceaux de renvois », qui sont loin de n'être qu'anecdotiques (Detrez, 2002 : 116).

C'est dans ce cadre que doit être envisagé ce qui est demandé aux nouveaux immigrants israéliens jusque dans les années 1970 : l'injonction de pratiquer les Rikoudéi Am plutôt que leurs propres répertoires dansés (Gibert, 2011). Ce travers de l'utopie sioniste mise en pratique, travers souligné par de nombreux historiens, qui consiste en un assujettissement symbolique des Juifs non européens aux cadres sociaux et culturels des "pionniers » venus d'Europe, passe alors par un assujettissement des corps et de leur reconstruction dans un moule unique et faussement neutre qui ne sera pas vraiment contesté avant la deuxième moitié $\mathrm{du} X \mathrm{X}^{\mathrm{e}}$ siècle.

\section{Faire autre chose. S'opposer à l'Autre}

En creux du processus précédent se lit l'autre versant de la dialectique: transformer les corps peut au contraire permettre de lutter contre les représentations en en faisant autre chose. 
Pour le sionisme, transformer les corps juifs, c'est aussi faire contre, et défaire, les images antisémites projetées sur ces $\operatorname{corps}^{48}$. Mais tandis que la nouvelle pratique dansée permet ainsi aux Juifs de se libérer de la force de destruction de ces stéréotypes, elle peut, comme nous venons de le voir, devenir à son tour aliénante pour celles et ceux qui souhaiteraient pratiquer d'autres formes dansées.

Ainsi, au tournant des années 1970, de nombreuses contestations s'élèvent des rangs des Juifs venus d'Afrique et d'Asie pour que la situation d'inégalité socio-économique qui double cette différence de valorisation des groupes constitutifs de la société israélienne soit modifiée. Un processus de relégitimation passe alors par la reconnaissance culturelle des pratiques apportées de diaspora par ces groupes, et l'un des moyens adopté passe par la transformation de la danse : sont crées des troupes de spectacle présentant des répertoires dansés issus de la diaspora, et en parallèle, des éléments géographiquement et culturellement identifiés comme appartenant aux Juifs venus de tel ou tel pays sont introduits dans les Rikoudéi Am. Ces transformations sont volontaires, et permettent de lutter contre le rouleau compresseur unificateur des premières décades. Pour autant, il ne s'agit pas de s'opposer radicalement à l'existence des Rikoudéi Am ni à la construction d'une identité israélienne qu'ils promeuvent, mais de mettre en lumière la dimension composite de la population d'Israël en juxtaposant différents répertoires plutôt que les substituer les uns aux autres (Gibert 2004, 2007a, 2008, 2011 ; Seroussi et Regev 2004).

Au-delà de ce "cas clinique » de la création des Rikoudéi Am, la littérature en sciences sociales montre que les phénomènes de mobilisation de la danse et/ou de la musique à des fins de construction identitaire et de rapports de pouvoir se retrouvent partout à travers le monde et à travers l'histoire, dans différentes configurations politiques, sociales, idéologiques, etc. (Kealiinohomoku 1998 [1969-70], Giurchescu 1991, Trebinjac 2000, Shay 2002, Buckland 2006, Leucci 2008). Il est donc central pour l'anthropologie de considérer les pratiques dansées comme point d'entrée fécond pour l'analyse de ces phénomènes, en envisageant la danse à la fois comme reflet d'une situation vécue, mais aussi et surtout, comme moyen d'action sur cette situation, lui permettant de la renforcer, ou au contraire de la contrer, voire de la modifier. Il devient alors plus pertinent d'analyser les pratiques dansées non tant dans leur dimension représentative - elles illustreraient quelque chose qui, d'une certaines manière, leur préexisterait - que dans leur dimension performative et générative : elles sont elles-mêmes un moyen de générer, de construire quelque chose: des corps (ceux des danseurs ou des spectateurs), des regards, des relations (de genre, de parenté, de pouvoir, de

48 D. Wildmann montre ainsi que les discussions publiques des gymnastes juifs européens au tournant du XXe siècle ont fréquemment recours aux couples d'antonymes tels que fort/faible (strong/weak), droit/tortueux (upright/crooked) ou encore lâche/ tendu (loose/taut) (2006 : 34). 
domination, de reconnaissance), de l'« identité » collective ou individuelle, de la revendication $\mathrm{d}^{\prime}$ " authenticité ", de l'exotisme, un remède à la nostalgie de l'exil, de l' « être ensemble », de la transmission de savoir, etc.

Le corollaire méthodologique de tout cela apparaît également de manière saillante : il est tout aussi nécessaire de travailler sur la matière dansée et l'expérience sensible elles-mêmes que sur le contexte, les parcours des protagonistes, etc. Cela demande d'analyser les transformations des corps, de la matière dansée, de son organisation (dans le temps, l'espace, le corporel) mais aussi d'interroger ce qui (dans les propriétés formelles de la danse et audelà) permet ces transformations et la prise en charge de multiples plans et/ou d'enjeux différents, voire contradictoires (plasticité, propriétés polymorphes et polysèmes de la matière, conjugaison de plans multiples en simultanéité, etc.). Doit également être posée la question des limites : qu'est-ce qui ne peut pas, ou ne peut plus être transformé ? Par qui, quand, comment et pourquoi ?

Ainsi, au-delà de cette étude de cas spécifique, c'est la puissance heuristique d'une étude anthropologique de la danse et des pratiques culturelles et corporelles en général à apporter un nouvel éclairage sur les processus de constructions identitaires et de rapports de pouvoirs que j'ai essayé de mettre en évidence ici. L'approche propose de tenir ensemble une analyse des contextes de production, de performance (spectaculaire ou non) et de réception de ces pratiques, une identification des différentes catégories d'acteurs impliqués et de leurs motivations, et une étude de la matière dansée elle-même et des traitements qu'elle subit, tout cela afin de saisir comment s'effectuent en pratique la mobilisation et le traitement de ces matériaux pour répondre aux divers enjeux analysés.

Passer par une triple analyse des pratiques dansées a permis d'aborder ces questions non seulement au niveau du dire mais aussi du faire - remaniement formel, critères de choix, et instrumentalisation des pratiques au nom des discours, des représentations et des (en)jeux de pouvoir, ici en contexte de migration et de déplacement de population.

\section{ÉPILOGUE}

"L'une des choses les plus dures pour les danseurs, ce fut de danser à l'unisson " explique le chorégraphe de danse contemporaine Yuval Pick à propos de sa pièce Folks, créée en France en 2012 avec des danseurs formés à la danse contemporaine ${ }^{49}$.

\footnotetext{
${ }^{49}$ Sources : discussions personnelles avec Yuval Pick ; débats publics en sa présence, et site du Centre Chorégraphique National de Rilleux-la-Pape (www.ccnr.fr). Les propos tenus dans cet article n'engagent cependant que moi, dans la mesure où ils n'ont pas été prononcés dans l'optique de cet article.
} 
Si homokinésie, homorythmie et mains tenues ne sont effectivement pas courantes dans la danse contemporaine actuelle ou dans les pratiques corporelles du quotidien - au moins dans la plupart des pays d'Europe et d'Amérique du Nord, ce sont au contraire deux éléments centraux des Rikudei Am à partir desquels Yuval Pick, né en Israël et ayant pratiqué cette forme de danse pendant son adolescence avant de se tourner vers la danse contemporaine, a débuté son travail de création artistique pour cette pièce intitulée Folks, avant de s'en éloigner à grands pas pour construire sa propre pièce $^{50}$. Lorsque l'on poursuit l'échange avec ce chorégraphe, émerge aussi la surprise qu'il a eue en revisitant une forme dansée qu'il n'avait pas pratiquée depuis des dizaines d'années: pensant y retrouver de la joie et de l'insouciance, il en vient à interroger la difficulté, au quotidien, tant pour les adolescents que pour les adultes, de se tenir par la main lorsque l'on ne partage pas des liens d'intimité très forte avec la ou les personnes à qui l'on donne la main. Ces deux points l'ont aussi amené à penser sa pièce autour de l'articulation entre groupe et individu, unisson et différences.

A leur manière, ces questions font écho à ce qui traverse aujourd'hui le monde des Rikudei Am et questionnent indirectement le résultat, soixante-dix ans plus tard (1944-2014), des processus analysés dans cet article.

En effet, si l'unisson et l'homokinésie sont encore l'une des composantes fondamentales des danses créées aujourd'hui dans les Rikoudei Am, en revanche, la volonté des premières créatrices de maintenir un contact permanent entre les danseurs (en se tenant par la main ou par les épaules par exemple) afin de garder l'unité recherchée, est largement remise en cause par la pratique actuelle des Rikoudei Am au cours de laquelle le contact physique est très rare, hormis lors des danses de couple.

Regrettant cette situation dans laquelle ils lisent «une perte d'authenticité », certains enseignants et organisateurs d'harkadot (soirées de pratique de danse israélienne) organisent depuis une dizaine d'années, outre les soirées dites « normales », des soirées qualifiées de «nostalgiques $»^{51}$, c' està-dire au cours desquelles seules les danses créées jusque dans les années 1980 sont exécutées, et où tenir par la main les autres danseurs est de rigueur.

50 Né en 1970 en Israël, danseur dans une troupe de Rikudéi Am pendant plusieurs années, puis formé en danse contemporaine à la Bat-Dor Dance School puis à la Batsheva Dance Company (Israël), ce chorégraphe vit en Europe depuis 1995, et plus particulièrement en France depuis 2002. La création chorégraphique dont il est question ici, Folks, a vu le jour en 2012 au Centre Chorégraphique National de Rilleux-la-Pape dont il est actuellement le directeur.

51 Ce type de soirée est devenu une catégorie à part entière de certains sites permettant d'identifier les lieux où danser en Israël (voir par exemple le site de l'association des danseurs de Rikoudéi Am: www.harokdim.org). Certaines de ces soirées sont baptisées pour l'occasion: «Danser comme autrefois» (Lirkod be ta'am shel Pa'am), "Soirée des roses» (Erev Shel Shoshanim), etc. 
Ce faisant, c'est le processus de normalisation de cette forme dansée qui est ainsi mis en relief. Les premières créatrices étaient en effet conscientes de créer quelque chose $\mathrm{d}^{\prime}$ " artificiel ${ }^{52}$, de «non-organique ${ }^{53}$, de « forcé ${ }^{54}$, et elles pensaient que le tri des «vraies danses » se ferait peu à peu, par leur résistance au temps qui passe, aux goûts des danseurs qui évoluent, au plaisir kinésique qu'éprouvent ceux qui les effectuent :

Quant aux «Rikoudei Am», il était évident pour tous, et surtout pour nous les gens de la commission, qu'il y avait de l'exagération dans cette appellation dont nous avions paré nos danses. Il était clair pour nous qu'elles n'étaient encore que des propositions, de l'espoir, des bourgeons de danse populaire (Rikoudei Am). Dans une ou deux générations seulement se purifieraient celles d'entre elles qui demeureraient vraiment des danses populaires (Kadman, 1969 : 17-18).

Et c'est ce qui semble s'être passé, puisque sur les milliers de danses créées pendant ces soixante-dix années, seules quelques centaines sont dansées régulièrement : certaines danses disparaissent des cours et soirées de pratique quelques mois seulement après leur création, tandis que d'autres au contraire perdurent et traversent les décades et les océans.

La greffe semble donc avoir pris : ce qui était «nouveau » est devenu «le vrai », "l'authentique». La transformation volontaire est devenue héritage ancestral, et le mythe fonctionne.

\section{BIBLIOGRAPHIE}

Michel ABITBOL, "La mémoire occultée et retrouvée: Juifs d'Orient et de Méditerranée en Israël », Lettre du Centre de Recherche Français de Jérusalem 12, 1995, pp. 41-49.

AHAD-HA-AM, Au carrefour, Trad. de l'hébreu [Al Parachat Dera'him], Textes choisis et traduits par A. Gottlieb, Paris, Lipschutz, 1938 [1894].

52 "We have to create our dances artificially until the time when there can be organic growth. Then the bad dances will drop away and the show-off quality will go; there'll be a combination of the new and the old with the native spirit from our land" (Y. Cohen, citée par Ingber, $1974: 36$ ).

53 "For people who fervently wished to have dances of our own and in our lifetime, it was clear that we had no choice: we had to create dances and this is what happened, starting in $1944 \ldots$ This was against all the laws of the development of folk culture the world over. How can one create purposely, artificially, folk dances which usually grow slowly like trees out of deep roots ... a process of hundreds years... but after all, the same artificial growth was true of the rebirth of the nation when immigrants from all over the world endeavored to built agriculture, industry, a state with a national culture and economy (Kadman, citée par Ingber, $1974: 9)$.

54 "Like everything else here - agriculture, education, construction - the making of this folk dance gathering was by force of will, not slow built by well-settled, established people whose country grew little by little with hundreds of years" (S. Levi-Tanai, citée par Ingber, $1974: 26$ ). 
Gaby ALDOR, «Agadati, Baruch », Dictionnaire de la Danse, Paris, Larousse, 1999, pp.3.

Anonyme, "Their Ballet is 2.000 Years Old", Illustrated, 3.4.1948.

Benedict ANDERSON, Imagined Communities, London, Verso, 1983.

John AUSTIN, Quand dire, c'est faire, Paris, Seuil, 1970 [Ed. or. How to do things with words. Oxford, Oxford University Press, 1962].

Frederic BARTH, Ethnic groups and boundaries. The social organisation of culture différence, Bergen-Oslo, Universitetsforlaget, 1969.

Daniel BOYARIN \& Jonathan BOYARIN, Pouvoirs de Diaspora. Essai sur la pertinence de la culture juive, Paris, Cerf, 2007 [2002].

Michael BRENNER \& Gideon REUVENI (dir.), Emancipation through Muscles. Jews and Sports in Europe, Lincoln and London, University of Nebraska Press, 2006.

Theresa J. BUCKLAND (dir.), Dancing from Past to Present. Nation, Culture, Identities. Madison, The University of Wisconsin Press, 2006.

Denis CHARBIT, «Consensus politique, modernisation étatique et culture démocratique à l'épreuve des clivages et tensions de la société israélienne », in Carol IANCU (dir.), Permanences et mutations dans la société israélienne, Montpellier, Université Paul Valéry-Centre de recherches et d'Etudes Juives et Hébraïques, 1996, pp. 137-170.

Denis CHARBIT, Sionismes. Textes fondamentaux, [Textes réunis et présentés par D. Charbit], Paris, Albin Michel, 1998.

Erik COHEN, "Ethnicity and Legitimation in Contemporary Israel", The Jerusalem Quarterly 28, 1983, pp. 111-124.

Christine DETREZ, La construction sociale du corps, Paris, Points Seuil, 2002.

Alain DIECKHOFF, "Sionisme et judaïsme : la difficile et fragile autonomie du politique ", Revue française de science politique, 39e année, n6, 1989, pp. 816-828.

Alain DIECKHOFF, L'invention d'une nation. Israël et la modernité politique. Paris, Gallimard, 1993.

Donna Robinson DIVINE, Exiled in the Homeland. Zionism and the Return to Mandate Palestine, Austin, University of Texas Press, 2009.

Mary DOUGLAS, De la souillure. Essai sur les notions de pollution et de tabou, Paris, La Découverte, 2001 [Ed. or. Purity and Danger. London, Routledge \& Kegan Paul Ltd, 1967].

Michel FOUCAULT, Surveiller et punir, Paris, Gallimard, 1975.

Zvi FRIEDHABER, “The development of Folk Dance in Israel”, Israel Dance 1987/88, pp. 33-39.

Jay GELLER, «Un avenir sans juifs : les écrits présionistes de Nordau », in Delphine BECHTEL, Dominique BOUREL et Jacques LE RIDER (dir.), Max Nordau. 1849-1923, Paris, Cerf, 1996, pp.225-243.

Ernest GELLNER, Nations and Nationalism, Oxford, Basil Blackwell, 1983.

Marie-Pierre GIBERT, “The intricacies of being Israeli and Yemenite. An Ethnographic Study of Yemenite "Ethnic" Dance Companies in Israel", Qualitative Sociology Review. III-3, December 2007a, pp. 100-112. 
Marie-Pierre GIBERT, «Les aventures du 'Pas Yéménite'. Mouvement dansé et constructions identitaires en Israël », 3e Congrès du Réseau Asie. Paris, France, 2007b [en ligne]. URL: http://www.reseau-asie.com/cgibin/prog/gateway.cgi?langue=fr\&password=\&email=\&dir=myfile_colloque\&type $=\mathrm{j}$ $\underline{\text { hg54gfd98gfd4fgd4gfdg\&id=422\&telecharge_now }=1 \& \text { file=a38gibert_mariepierre.pdf }}$

Marie-Pierre GIBERT, «Le henné est-il encore efficace? Faux mariés et vrais Yéménites dans les spectacles des troupes de danse "ethniques » en Israël », in Marie-Luce GÉLARD (dir.), Les usages du henné: pratiques, rites et représentations symboliques, Paris, Presses Universitaires de Nancy, 2008, pp. 21-52.

Marie-Pierre GIBERT, «La construction d'un 'Yéménite israélien' par la danse », in Marie-Christine BORNES VAROL (éd.), Chocs de langues et de cultures ? Un discours de la méthode, Presses Universitaire de Vincennes, 2011, pp. 347-393.

Sander GILMAN, The Jew's Body, London-New York, Routledge, 1991.

Anca GIURCHESCU, "The Use of Traditional Symbols for Recasting the Present : A case Study of Tourism in Rumania", Dance Studies 14, 1991, pp. 47-63.

Jack R. GOODY, The Myth of the Bagre, Oxford, Oxford University Press, 1972.

Theodor HERZL, Altneuland, 1902 [Trad. fr. Terre ancienne terre nouvelle..., Paris, Editions Rieder, 1931].

Jehoash HIRSHBERG, "Performers between East and West - Ideology and Reality in the Yishuv", in Amnon SHILOAH (dir.), The Performance of Jewish and Arab music in Israel today. Part 1. Amsterdam, Harwood Academic Publishers, 1997, pp. 5-13.

Eric HOBSBAWM \& Terence RANGER (dir.), The Invention of Tradition, Cambridge, Cambridge University Press, 1983.

Judith INGBER BRIN, "Shorashim: The Roots of Israeli Folk Dance", Dance Perspectives 59. 1974, pp. 1-59.

Judith INGBER BRIN, “Vilified or glorified? Views of the Jewish body in 1947”, Jewish Folklore and Ethnology Review, 20 (1-2), 2000, pp. 39-58.

Judith INGBER BRIN, Seeing Israeli and Jewish Dance, Detroit, Wayne State University Press, 2011.

Julius ISAAC, "Israel - A New Melting Pot?", in Wilfried David BORRIE (dir.), Cultural Integration of Immigrants, Paris, Unesco, 1959, pp. 234-266.

François JULLIEN, Les Transformations silencieuses, Paris, Grasset, 2009.

Gurit KADMAN, "Yemenite Dances and their influence on the New Israeli Folk Dances", International Folk Music Journal 4, 1952, pp. 27-30.

Gurit KADMAN, Am Roked [Une Nation qui danse], Jérusalem-Tel Aviv, Ed. Shoken [en hébreu], 1969.

Gurit KADMAN, "Tradition and creation in Israeli Folk Dances", in Pamela SQUIRES, Selected Lectures from "Introduction to Folklore (Dance) of Israel", The Hebrew University of Jerusalem, Folklore Research Center-Overseas Students of Summer Course, 1972. [Document non publié ; Archives Z. Friedhaber - Bibliothèque de la Danse, Tel Aviv].

Elke KASCHL, "Beyond the Nation in Israeli Folk Dancing. Performing Community in New York under the Impact of Globalization", Jewish Folk \& Ethnology Review 20 (12), 2000, pp. 59-77. 
Elke KASCHL, Dance and Authenticity in Israel and Palestine. Performing the Nation, Leiden-Boston, E.J. Brill, 2003.

Joann KEALIINOHOMOKU, « Une anthropologue regarde le ballet classique comme une forme de danse ethnique », Nouvelles de Danse, 34-35, 1998, pp.47-67 [éd. or. in Impulse, 1969-70].

Isabelle LACOUE-LABARTHE, Femmes, féminisme, sionisme dans la communauté juive de Palestine avant 1948, Paris, L'Harmattan, 2012.

Gérard LENCLUD, «La tradition n'est plus ce qu'elle était... », Terrain 9, Octobre 1987.

Gérard LENCLUD, "Qu'est-ce que la tradition? », in Marcel DETIENNE (dir.), Transcrire les mythologies. Tradition, écriture, historicité, Paris, Albin Michel, 1994.

Tiziana LEUCCI, «L'apprentissage de la danse en Inde du Sud et ses transf" rmati ns au XXème siècle : le cas des devadāsī, rājadāsī et națuvan̄ār », Rivista di Studi Sudasiatici, 2008/III, pp.49-83.

Giora MANOR, “The Legend of Agadati”, Israel Dance, 1976, pp. 8-10.

Joseph MICHMAN, Cultural policy in Israël, Paris, Unesco, 1973.

George L. MOSSE, L'Image de l'homme. L'invention de la virilité moderne, Paris, Editions Abbeville, 1997 [ed. originale en anglais 1996].

George L. MOSSE. «Max Nordau, le libéralisme et le «nouveau juif » », in Delphine BECHTEL, Dominique BOUREL et Jacques LE RIDER (dir.), Max Nordau. 1849-1923. Paris, Cerf, 1996, pp.11-29.

Max NORDAU, « Muskeljudentum », Jüdische Turn-Zeitung, June 1900.

Max NORDAU, Ecrits Sionistes, Paris, Lipschitz, 1936 [Ed. or. Zionistiche Schriften, Köln, 1909].

Max NORDAU, Dégénérescence, Paris, F. Alcan, 1894 [Ed. or. Entartung. Berlin, Duncker u. Humbolt, vol.1 -1892, vol. 2 -1893].

Mona OZOUF, L’Homme régénéré. Essai sur la Révolution française, Paris, Gallimard, 1989.

Tudor PARFITT, The road to redemption. The Jews of Yemen 1900-1950, Leiden-New York-Köln, E.J. Brill, 1996.

Raphael PATAI, On culture contact and its working in modern Palestine, Menasha, Wisc., American Anthropological Association, 1947.

Jean POUILLON, «Tradition: transmission ou reconstruction?», in Jean POUILLON, Fétiches sans fétichisme, Paris, F. Maspero, 1975.

Jean POUILLON, «Plus c'est la même chose, plus ça change », in Jean POUILLON, Le cru et le su, Paris, Seuil, 1993.

Jean POUILLON, "Appartenance et identité », in Jean POUILLON, Le cru et le su, Paris, Seuil, 1993.

Todd PRESNER, Muscle Judaism : The Jewish Body and the Politics of Regeneration, New York, Routledge, 2007.

Motti REGEV \& Edwin SEROUSSI, Popular Music \& National Culture in Israel, BekeleyLos Angeles-London, University of California Press, 2004. 
Ernest RENAN, Qu'est-ce qu'une nation?, Paris, Presses Pocket, 1992. [Conférence prononcée à la Sorbonne le 11 mars 1882].

Dan RONEN, «1944-1994. Fifty Years of Israeli Folk Dance », Israel Dance, 1994, pp. 121-123.

Tom SEGEV, Les Premiers Israéliens, Paris, Calmann-Levy, 1998 [Ed. or. 1949 : HaIsraelim Ha-Rishonim, 1984 (en hébreu)].

Yaacov SHAVIT \& Shoshana SITTON, Staging and Stagers in Modern Jewish Palestine. The Creation of Festive Lore in A New Culture, 1882-1948, Detroit, Wayne State University Press, 2004.

Anthony SHAY, "Parallel Traditions : State Folk Dance Ensembles and Folk Dance in "The Field"”, Dance Research Journal 31(1), spring 1999, pp. 29-56.

Anthony SHAY, Choreographic Politics. State Folk Dance Companies, Representation and Power, Middletown Co., Wesleyan University Press, 2002.

Nina S. SPIEGEL, "Cultural formulation in Eretz Israel: the National Dance Competition of 1937", Jewish Folklore and Ethnology Review, 20 (1-2), 2000, pp. 24-38.

Nina S. SPIEGEL, Embodying Hebrew Culture. Aesthetics, Athletics, and Dance in the Jewish Community of Mandate Palestine, Detroit, Wayne State University Press, 2013.

Zeev STERNHELL, The Founding Myths of Israel : Nationalism, Socialism and the Making of the Jewish State, Princeton, Princeton University Press, 1999.

Anne-Marie THIESSE, La création des identités nationales. Europe XVIIIe-XIXe siècle, Paris, Points Seuil, 1999.

Sabine TREBINJAC, Le pouvoir en chantant. T.1. L'art de fabriquer une musique chinoise, Société d'Ethnologie, Nanterre, 2000.

Jacques ULMAN, De la gymnastique aux sports modernes, Paris, PUF, 1965.

George VIGARELLO, Le corps redressé, Paris, J-P Delarge, 1978.

Daniel WILDMANN, "Jewish Gymnasts and Their Corporeal Utopias in Imperial Germany", in Michael BRENNER \& Gideon REUVENI (dir.), Emancipation through Muscles. Jews and Sports in Europe, Lincoln and London, University of Nebraska Press, 2006, pp.27-43.

Yael ZERUBAVEL, "The historic, the legendary, and the incredible: invented tradition and collective memory in Israel", in John R. GILLIS (dir.), Commemorations. The Politics of National Identity, Princeton, Princeton University Press, 1994, pp.105123.

Yael ZERUBAVEL, Recovered Roots : Collective Memory and the Making of Israeli National Tradition, Chicago, University of Chicago Press, 1995.

Yael ZERUBAVEL, «Le héros national: un monument collectif. Traditions et politiques de commémoration. Exemples israéliens », in Pierre CENTLIVRES, Daniel FABRE et Françoise ZONABEND (dir.), La Fabrique des héros, Paris, Editions de la Maison des Sciences de l'Homme, 1998, pp. 167-179.

Moshe ZIMMERMAN, "Muscle Jews versus Nervous Jews”, in Michael BRENNER \& Gideon REUVENI (dir.), Emancipation through Muscles. Jews and Sports in Europe, Lincoln and London, University of Nebraska Press, 2006, 
RÉSUMÉ : Née officiellement en 1944, inconnue du grand public en dehors d'Israël quoique largement pratiquée par des Juifs et des non-Juifs tant en Israël que dans plus de trente pays, de l'Asie à l'Europe en passant par les Amériques, s'enrichissant tous les ans de plusieurs centaines de nouvelles danses, la danse populaire israélienne (Rikoudéi Am en hébreu, littéralement "les danses du peuple») semble toujours bien vivante en ce début du XXIe siècle. Mais de quoi s'agit-il exactement ? Et pourquoi parler de cette forme dansée, ici, dans un numéro portant sur les présences au corps, dans une section sur l'immigration? En quoi l'analyse de la création et du développement d'une pratique dansée spécifique peut-elle intéresser l'anthropologie sociale de manière large ? La réponse suit trois fils qui vont se nouer au cours de cet article : analyse quasi clinique de la construction d'un État-nation à travers l'élaboration d'une pratique corporelle; réflexion sur le rapport au corps et l'incorporation; question des modes «d'intégration » des migrants dans une nouvelle société. La (re)construction d'un État israélien dès les premières décades du XXe siècle s'accompagne de la volonté de «normalisation » de cet État-nation en devenir, selon les critères des nationalismes européens de l'époque: un peuple, une terre, une langue, une culture. Or les Juifs ayant vécu plusieurs siècles dans des environnements socio-culturels extrêmement divers, cette construction nationale doit passer par la création d'une culture unique qui permettrait d'unifier ces différences. Cette normalisation de l'État-nation se loge en outre dans l'élaboration d'un "Homme Nouveau », ou « Nouvel Hébreu », un juif " régénéré » dont le corps serait façonné pour répondre aux différents enjeux mis en avant par les pensées sionistes de l'époque : lutte contre les stéréotypes antisémites, construction/ "renaissance » d'un pays, unification d'une nation, etc. Combinant ces deux attentes du sionisme (culture nouvelle et transformation des corps), se crée une nouvelle pratique dansée, forme unique, unissant les citoyens de l'État-nation en devenir, et gommant les disparités issues des siècles vécus en diaspora aux quatre coins du monde. Revenir sur l'histoire de la création et du développement de cette forme dansée jusqu'à la situation actuelle permettra d'envisager sous un jour nouveau la manière dont la construction du corps humain selon un modèle spécifique est au cœur d'enjeux politiques allant bien audelà de la « simple » question des pratiques artistiques.

MOTS-CLÉS : Corps, danse, Homme Nouveau, Israël, migration, nation, régénération. 